\title{
Editorial
}

\section{Triple negative breast cancer: Breast cancer research in evolution}

\author{
Jennifer Eng-Wong ${ }^{\mathrm{a}, *}$, Sally Hunsberger ${ }^{\mathrm{b}}$ and Jo Anne Zujewski ${ }^{\mathrm{c}}$ \\ ${ }^{a}$ Lombardi Comprehensive Cancer Center, Georgetown University, Washington DC, USA \\ ${ }^{\mathrm{b}}$ Biometrics Research Branch, Division of Cancer Treatment and Diagnosis, National Cancer Institute, Bethesda \\ MD, USA \\ ${ }^{\mathrm{c}}$ Clinical Trial Evaluation Program (CTEP), National Cancer Institute, Bethesda MD, USA
}

\begin{abstract}
A 2011 Pubmed search on the terms "breast cancer" and "triple negative" identified 932 articles, thus interest in this topic since the classification of triple negative/basal-like breast cancer has exploded and a number of recent informative reviews have been published [1-3]. We envision this special edition of Breast Disease focused on triple negative breast cancer (TNBC) as a "go-to" handbook accessible to clini$\mathrm{cal}$, translational and laboratory researchers in designing (and interpreting) studies of triple negative breast cancer. This issue contains in-depth reviews of multiple aspects of TNBC from triple negative cell lines to current clinical trials. We, the editors, would like to thank the authors for their excellent contributions and highlight a number of over arching issues.
\end{abstract}

\section{The definition of triple negative breast cancer is evolving}

The term 'triple negative' is short hand for estrogen receptor (ER) negative, progesterone receptor (PR) negative and HER-2 neu negative breast cancer. It is closely linked to, but not synonymous with, "basallike" breast cancer defined by gene array analysis. "Basal like" refers to the presence of basal or epithelial cell markers, including cytokeratins 5/6, 14 and 17 [4]. $\mathrm{Ma}$ et al. showed the heterogeneity of "triple negative" breast cancer using gene expression profiling, and

\footnotetext{
*Corresponding author. E-mail: je95@georgetown.edu.
}

TNBC includes basal-like, claudin-low, apocrine and HER2 enriched subtypes. Laboratory research continues to finely hone the classification of TNBC. Thus although we continue to think of these disease entities as interchangeable, they are indeed distinct [5,6].

In the laboratory, a wealth of viable options exist to model TNBC. Ironically the clinically aggressive nature of TNBC lends itself to useful laboratory features: relative ease of growth of TNBC cell lines and robust animal models for experimentation. Both cell lines and animal models provide relevant and accessible tools. TNBC cell lines mirror the original tumors morphologically and by cDNA gene expression analysis (Chavez et al.); animal models offer multiple ways to manipulate tumors to gain a better understanding of the molecular drivers of TNBC and the role of BRCA mutations (Herschkowitz et al., Diaz-Cruz et al.). The application of clinical care and specific treatment recommendations to the larger category of TNBC, much less the newly defined TNBC sub-types, is still undefined. Ensuring that these newly defined diseases retain clinical relevance is a current challenge. Present needs include real time categorization of disease that extends beyond the scope of immunohistochemical (IHC) testing. Additionally an understanding of the critical pathways that translate tumor "type" to "target" are required. A major research challenge of the next few decades will be to further define subtypes within the major "surrogate" groups and develop targeted therapies for these subtypes. 
Who gets triple negative breast cancer?

TNBC is more common in younger women and younger African American women. Gierach et al. nicely summarize what is known regarding the epidemiology of TNBC, and Peshkin et al. review the overlay of BRCA mutation status and triple negative breast cancer. Both authors note that there are numerous areas of missing data, e.g. the SEER database has not collected HER2 status and there is little data on ethnicity, BRCA mutations and cancer sub-type. Thus it is clear that we need to start collecting epidemiology data in a more disciplined manner with all tumor characteristics duly noted. Of course fully annotated tissue banks would be incredibly informative and increasingly tissue is requested on trial, but the mechanisms for maintaining these banks remain complicated and therefore risky. At this time the associated risk factors are reported in relatively small sample sizes which should invoke caution on the part of investigators now planning primary or secondary prevention interventions. We also wish to point out that although as physicians and scientists we have adopted the term "triple negative" it is important to consider this term from a patient perspective. "Triple negative" implies a certain degree of pessimism that is not lost on patients. "Basal like" may be a more acceptable alternative, although we recognize that this is technically a specific subtype of TNBC.

\section{How should we treat triple negative breast cancer?}

We are now faced with the challenge of moving the elegant characterization of tumors in a research lab to the "real world." The proposed models of the evolution of TNBC from mammary stem cells is well described by Ganesan et al. and opens multiple lines of inquiry into the potential treatment and prevention of TNBC. In the inclusive review by Rodler et al. it is clear that standard chemotherapy agents (anthracyclines, taxanes) are active in TNBC and that more chemotherapy, i.e. addition of taxanes following anthracycline therapy is better. There is limited data on other agents (capecitabine, platinum agents) and multiple studies are ongoing to address these and newer targeted agents. It is important to note that nearly all analyses of standard chemotherapy agents in TNBC are post-hoc analyses and thus hypothesis generating. We are just starting to evaluate TNBC as a distinct disease in tailored trials with much of the focus on evaluating platinum agents and PARP inhibitors (see Table 2 Reeder Hayes et al.). The PARP inhibitor story is a complex one. There are significant pre-clinical and clinical differences in the major PARP compounds in development. Since these reviews were written, Sanofi-Aventis has made public the results of their phase 3 trial of iniparib (BSI-201) in metastatic TNBC results of which are expected to be presented at a major meeting in 2011. Disappointingly, the trial was negative, although a pre-planned secondary analysis of the treatment in the second and third line setting confirmed the randomized phase 2 study in previously treated patients [7]. As these reviews point out the designation of TNBC by IHC is already an oversimplification of the disease yet this is the most clinically accessible classification currently available. Newer clinic-friendly assays are being developed but are not yet commonly employed [8]. Defining TNBC and its subtypes has further implications for clinical trial design.

\section{How should clinical trial design address TNBC?}

While the identification of multiple subtypes of breast cancer has clearly demonstrated that "lumping" different subtypes of breast cancer together is unwise due to differing biology and prognosis, we are now faced with the opposing problem. Currently there are 13 trials in the metastatic setting and 18 in the neoadjuvant or adjuvant setting. Limiting our sights to the (neo) adjuvant trial setting only, overall accrual goals total more than 7500 participants (based on Reeder Hayes article). By back-of-the-napkin math, if $20 \%$ of new breast cancers in the U.S. are TN and approximately $3 \%$ of cancer patients participate in clinical trials, approximately 1254 women annually would enroll in the U.S. in these studies. Thus it is clear that collaborative international research is imperative to address this disease. If we further dissect TNBC into sub-categories these diseases will only become more challenging to study. To this end, a few comments on eligibility and endpoints merit discussion that may seem counter-intuitive but offer a realistic approach.

\section{Selection of patients}

ASCO-CAP guidelines recommend that tumors with less than $1 \%$ cell staining for ER or PR (by IHC) are defined as ER or PR negative [9]. This was done for the sensible reason that there are data to show that women with even a small amount of ER positivity in their tumors can benefit from tamoxifen in the adjuvant setting [10]. Indeed one would want to use such an effective and low risk agent if there was a chance of benefit. However in the drug development field, this 
may result in excluding the very low ER expressing tumors from the study of agents that may very well be appropriate in a low ER setting. A practical approach to this problem is to adopt the ASCO-CAP definition of ER negative for the subjects selected for the primary analysis, but allow the ER/PR 1-9\% positive patients to enroll as a separate cohort. A secondary objective of the study would be an analysis of the entire population. Although the ER/PR 1-9\% positive cohort will not have adequate power for drawing firm conclusions, some hint can be obtained from the response of this group as opposed to the study as a whole. We have fondly called this approach "triple negative and friends". Of note basal-like breast cancer includes ER negative and ER low expression [11]. Similarly, some patients with equivocal HER2 testing can be included. ASCO-CAP guidelines state that the HER-2 FISH ratio of 1.8-2.2 is equivocal. Since HER-2 directed therapy may be offered to patients with a HER-2 ratio above 2 , allowing patients to enroll on a TNBC trial with a ratio of less than 2.0 can be considered.

\section{Clinical trial outcomes}

There has been a trend to evaluate "clinical benefit rate" (CBR) in phase 2 trials in metastatic breast cancer (MBC). It has been defined as complete response (CR) plus partial response (PR) plus stable disease (SD). However, CBR is a relatively new phenomenon, and it is hard to interpret results in early phase trials, as there are not adequate historical controls. A nice review of the need for randomized phase II/III clinical trials in cancer was published in Journal of Clinical Oncology [12] and we wholeheartedly agree with this. A single arm study of a new agent administered in combination with chemotherapy in breast cancer is unlikely to be informative. We also question the utility of the widespread adoption of CBR as an endpoint in phase 2 studies. The definition of SD has varied in breast cancer from 12 weeks to 6 months. In addition, the CBR would include patients who initially respond to therapy and then have progressive disease- this, arguably, would not be of "clinical benefit". In reporting phase 2 trials it would be preferable to report the response rate (CR plus PR), the per cent of patients who have not progressed at landmark analysis of 6 months and 12 months, and the progression free survival (PFS). We are very much supportive of efforts to standardize endpoints in trials of recurrent and MBC, much as was done by Hudis et al. in the adjuvant setting [13]. If an agent is not expected to cause tumor shrinkage, a randomized study is essential in order to screen for ac- tivity. Realistically, randomizing subjects to a targeted agent versus no therapy is impractical; these agents can be combined logically, but a control arm is necessary.

Although overall survival (OS) is the primary endpoint of interest to patients there has been a move towards using PFS as the primary endpoint in phase 3 trials. An endpoint other than OS in a phase 3 study should only be considered when death occurs many years after progression and there are active agents that will prolong survival after progression. In this situation PFS can be used as the endpoint since subsequent active therapies may dilute the ability to detect an initial benefit from the experimental agent. Others have argued that even other active agents are available as subsequent therapy for patients which might obscure detection of an overall survival benefit; this is the reality of the clinical situation and overall survival should be the primary endpoint (Korn and Abrams JCO 2011 in press). Unfortunately, TNBC as a distinct type of breast cancer has a grim prognosis. These patients are getting more therapies in the adjuvant setting, and have recurred following polychemotherapy with multiple agents without extending survival. The median PFS in patients with recurrent TNBC is 3-6 months and OS is only 6-9 months $[7,14,15]$. In this situation, OS is the preferred endpoint of clinical trials.

To illustrate these concepts at work consider this example. A randomized phase 3 study with a control arm and an experimental arm with OS as the primary endpoint would require 288 patients using a 1 -sided .025 level test with $90 \%$ power to detect a difference in median OS from 9 to 13.5 months (hazard ratio in of 1.5). In this design we assume an accrual rate of 10 patients per month and a minimum follow up of 2 years. Since this is a small patient population it may be useful to consider a phase II/III design where patients are accrued to the study, and an interim analysis is performed on PFS. If there is no evidence of activity the study will be stopped. If there is evidence of activity the study will continue to the overall sample size with OS as the primary endpoint. All patients would be used in the final analysis. Under the null situation of no benefit on PFS this can reduce the overall sample size. For example in the phase 3 study discussed above after 128 patients have been accrued to the study a comparison of PFS between the two groups would be performed. If the 1 -sided p-value is less than 0.3 the study would continue to the total sample size of 288 . The p-value of 0.3 and sample size at the interim analysis was chosen to maintain high overall power for the study but stop the study early if no evidence of activity was observed. 
This combination of parameters provides the smallest expected sample size when the null hypothesis of no benefit from the experimental treatment on PFS is true.

In summary although TNBC is a clinically distressing diagnosis, from the perspective of the scientific community the opportunities to better understand and ultimately affect outcomes in this subset of breast cancers is vast. Every level of scientific inquiry is critical, from determining the molecular and environmental triggers that lead to the development of TNBC to identifying factors implicated in growth, metastases and drug resistance. Finally understanding the clinical course of the disease is essential to appropriate clinical trial design.

\section{References}

[1] L. Carey, E. Winer, G. Viale, D. Cameron and L. Gianni, Triple-negative breast cancer: disease entity or title of convenience? Nat Rev Clin Oncol 7(12), 683-692.

[2] S.C. Linn and L.J. Van 't Veer, Clinical relevance of the triplenegative breast cancer concept: genetic basis and clinical utility of the concept, Eur J Cancer 45(Suppl 1) (2009), 11-26.

[3] M.D. Seal and S.K. Chia, What is the difference between triple-negative and basal breast cancers? Cancer J 16(1), 1216.

[4] C.M. Perou, T. Sorlie and M.B. Eisen et al., Molecular portraits of human breast tumours, Nature 406(6797) (2000), 747-752.

[5] L.A. Carey, C.M. Perou and C.A. Livasy et al., Race, breast cancer subtypes, and survival in the Carolina Breast Cancer Study, Jama 295(21) (2006), 2492-2502.

[6] B. Ghabach, W.F. Anderson, R.E. Curtis, M.M. Huycke, J.A. Lavigne and G.M. Dores, Adenoid cystic carcinoma of the breast in the United States (1977 to 2006): a population-based cohort study, Breast Cancer Res 12(4), R54.

[7] J. O'Shaughnessy, C. Osborne, J.E. Pippen et al., Iniparib plus chemotherapy in metastatic triple-negative breast cancer, $N$ Engl J Med 364(3) (2011), 205-214.

[8] J.S. Parker, M. Mullins and M.C. Cheang et al., Supervised risk predictor of breast cancer based on intrinsic subtypes, $J$ Clin Oncol 27(8) (2009), 1160-1167.

[9] M.E.H. Hammond, D.F. Hayes, M. Dowsett et al., American Society of Clinical Oncology/College of American Pathologists Guideline Recommendations for Immunohistochemical Testing of Estrogen and Progesterone Receptors in Breast Cancer (Unabridged Version), Archives of Pathology and Laboratory Medicine 134(7) (2010), E48-E72.

[10] R.M. Elledge, S. Green, R. Pugh et al., Estrogen receptor (ER) and progesterone receptor (PgR), by ligand-binding assay compared with ER, PgR and $\mathrm{pS} 2$, by immuno-histochemistry in predicting response to tamoxifen in metastatic breast cancer: a Southwest Oncology Group Study, Int J Cancer 89(2) (2000), 111-117.

[11] T. Sorlie, C.M. Perou and R. Tibshirani et al., Gene expression patterns of breast carcinomas distinguish tumor subclasses with clinical implications, Proc Natl Acad Sci U S A 98(19) (2001), 10869-10874.

[12] L.V. Rubinstein, E.L. Korn, B. Freidlin, S. Hunsberger, S.P. Ivy and M.A. Smith, Design issues of randomized phase II trials and a proposal for phase II screening trials, J Clin Oncol 23(28) (2005), 7199-7206.

[13] C.A. Hudis, W.E. Barlow and J.P. Costantino et al., Proposal for standardized definitions for efficacy end points in adjuvant breast cancer trials: the STEEP system, J Clin Oncol 25(15) (2007), 2127-2132.

[14] L.N. Harris, G. Broadwater, N.U. Lin et al., Molecular subtypes of breast cancer in relation to paclitaxel response and outcomes in women with metastatic disease: results from CALGB 9342, Breast Cancer Res 8(6) (2006), R66.

[15] F.B. Korn EL and J.S. Abrams, Overall survival as the outcome for randomized clinical trials with effective subsequent therapies, Journal of Clinical Oncology 2011, (in press). 